\title{
La chanson bâtarde de Stromae et Abd al Malik : Belgique, Afrique et francophonie
}

\author{
Stéphane HIRSCHI (Valenciennes) ${ }^{1}$
}

\section{Summary}

Since its beginnings, French chanson has been full of mixed musical influences. Two present-day French singers, Abd Al Malik and Stromae, preserve this tradition and renew it the same time. While singing about 'elsewhere' used to be a pretext for exoticism with commercial intentions, these two singers symbolise a generation where human mixity has become a major reality, which can also be observed in their songs. When Abd Al Malik, for instance, uses the pattern and inspiration of Jacques Brel's "Amsterdam" for his own song "Gibraltar", he changes both meaning and dynamics of the original song. Amsterdam's port is a tearing dead end ; Abd Al Malik's port is a place of transfiguration where you can 'sublimize' yourself and transform travelling into a productive initiation. On the same level, Stromae, metis and orphan himself, succeeds in sublimizing his pain in a dynamic danse with its success "Papaoutai" ; and he also succeeds in doing so in his song "Bâtard", a true art poétique, where thanks to his own maestro's abilities he presents hybridity as riches to be shared.

\section{Introduction : méthodologie et chronologie}

Le présent article entend aborder les représentations actuelles des formes de métissage dans la chanson francophone, autour de deux figures représentatives des chanteurs issus de l'immigration post-coloniale, en France et en Belgique : Stromae et Abd al Malik. On se placera dans une perspective cantologique consistant à envisager la forme chanson dans sa globalité sémantico-esthétique, articulant texte, musique et interprétation, conformément à mes précédents travaux présentés en bibliographie. Mais avant de considérer ces productions contemporaines, un balayage chronologique s'avère nécessaire, de façon à faire ressortir les évolutions actuelles dans ce traitement du métissage lyrique dans la chanson francophone. 
Le métissage a toujours été à l'œuvre dans la chanson française. Elle s'est toujours nourrie de rythmes et de sonorités venus de l'étranger. Déjà les troubadours mêlaient l'inspiration musicale du chant grégorien à un imaginaire lyrique venu du monde arabo-musulman. Aux débuts de l'enregistrement, le recours aux couleurs musicales exotiques était encore d'actualité, sur fond désormais d'Empire colonial. Dans le succès de Mayol en 1899, "À la cabane bambou ", la syntaxe fautive est supposée imiter un Africain :

\section{Moi, bon nègre tout noir, tout noir,}

[...] Mais moi bien trompé, toujours m'ennuyer,

Aussi, gros chagrin, moi le dire à vous,

Vouloir retourner chez nous.

À la cabane bambou bambou,

À la cabane bambou you!

Le refrain joue musicalement de l'onomatopée " you », partant dans les aigus, pour évoquer à la fois la supposée ‘ sauvagerie > du personnage, et scander une danse complice, destinée à faire rire. Le processus de collage de clichés prétendument exotiques se retrouve dans un autre succès, « Ma petite Tonkinoise », créée par Polin en 1906, dont les paroles choqueraient aujourd'hui :

Au Tonkin je suis parti

[...] Et je suis dev'nu l'chéri

D'une petit' femm' du pays

Qui s'appelle Melaoli

Ce paradis d'un Pigalle exotique, où les consonances mélanésiennes d'un nom n'abusent personne (Mélaoli !), nous propose un univers peuplé de femmes-objets aussi soumises qu'un pays colonisé est censé en fournir. L'exotisme habille ici le comique troupier. En 1931, Joséphine Baker reprend ce succès. Noire et américaine, figure d'un ailleurs, l'époque la trouve du coup crédible en Tonkinoise - pourtant une asiatique, mais si lointaine :

C'est moi qui suis sa petite

Son ananas, son ananas, son Annamite

Je suis vive je suis charmante

J'suis comme un z'oiseau qui chante

Il m'appelle sa p'tit'bourgeoise

Sa Tonkiki, sa Tonkiki, sa Tonkinoise

Le jeu des répétitions au refrain accentue cette confusion de tous les exotismes de l'Empire français. Joséphine Baker accepte d'incarner "Son ananas, son ananas, son Annamite ». Le 
jeu de mots permet l'association imaginaire : la femme-objet se métamorphose en fruit, belle à croquer, multicolore et féérique, à la fois noire et jaune, bariolage fantasmatique qui supprime les frontières mais toujours colonisée et donc possédée. L'instinct de domination de l'auditeur mâle est stimulé, mais édulcoré par l'air rassurant et accrocheur de Vincent Scotto, grand compositeur d'opérettes, un genre bien français.

Après-guerre, les couleurs changeront, méditerranéennes ou latines, mais le recours aux clichés exotiques permettra le même type de succès faussement étrangers pour Luis Mariano, Dalida, ou pour les rumbas artificielles d'un Tino Rossi et sa voix d'or au velours corse.

Quant aux influences de la culture anglophone, il suffit de savoir qu'un des premiers tubes de la scène française de la fin du XIX siècle, "Tha-ma-ra-boum-di-hé » n'est pas autre chose qu'une adaptation d'un succès anglais, pour comprendre l'importance des échanges rythmiques et mélodiques entre ces deux sphères culturelles. Il est donc logique que, dès la fin des années 30, la chanson française s'ouvre aussi aux influences du jazz. Avec Trenet, Vian ou Nougaro, ces nouveaux rythmes et instruments vont au fil des décennies traduire l'énergie d'une jeunesse dégagée des stéréotypes, comme dans ce dialogue des styles de 1962, «Le jazz et la java»:

Quand j'écoute béat un solo de batterie

V'là la java qui râle au nom de la patrie

Mais quand je crie bravo à l'accordéoniste

C'est le jazz qui m'engueule me traitant de raciste

Face à cette opposition initiale, la fin de la chanson valorise le croisement des deux traditions :

Jazz et java copains ça doit pouvoir se faire

Sur fond désormais de décolonisation, le recours à des couleurs rythmiques et instrumentales venues de l'étranger ne signifie plus alors un vernis extérieur et touristique, mais un véritable dialogue, un système d'échange qu'on désigne en tant que métissage. Claude Nougaro toujours, Pierre Vassiliu, adaptent des succès brésiliens de bossa nova ("Qui c'est celuilà », d'après Chico Buarque). Serge Gainsbourg se fait adepte du reggae en revisitant " La Marseillaise " sous le titre "Aux armes et cætera ». Et bien d'autres titres ou artistes nourris par cet échange incessant pourraient être cités, avant d'aborder les rivages métissés d'une bâtardise artistiquement revendiquée dans la chanson contemporaine...

Pourtant, après ce rapide aperçu historique, il me semble opportun de procéder encore à un double détour, déplacement spatial et retour temporel, à même de poser les enjeux esthétiques du métissage, par-delà les modes et les formes mis en avant selon les époques. Il va s'agir d'abord d'entendre l'espace de la chanson "Amsterdam » de Brel comme une représentation elle-même essentiellement composite, fort éloignée d'un réalisme de représentation. 
Ferré avait d'ailleurs bien entendu cette dimension fictive en proposant de peindre luimême vraiment un port des Pays-Bas, avec sa chanson « Rotterdam ».

De fait, musicalement, la mélodie de Brel est structurellement nourrie d'un fameux air élisabethain, "Greensleeves ", dont une écoute juste avant celle d' " Amsterdam » ne laisse aucun doute sur l'inspiration qui a nourri, inconsciemment ou non, la mélodie brélienne. Musicalement, «Amsterdam " place du coup naturellement l'auditeur dans un espace-temps élargi par rapport à un décor réaliste. Au lieu de l'imaginaire popularisé en chanson par les décors de Carco, Caussimon ou Mac Orlan et si bien relayés par Piaf ou Ferré, de la "Chanson de Margaret " à "Comme à Ostende " par exemple, cet univers de filles battues par le destin et de noirceur tragique, Brel impose une musique subtilement dansante, un rythme ternaire en 6/4, qui crée un arrière-plan transhistorique et universel, où la Renaissance chante encore aujourd'hui, et où la mer du Nord est abolie entre l'Angleterre et les Pays-Bas... Un espace-temps d'avant le port réel. Le jeu de l'imaginaire qu'instaurent les paroles de Brel conforte cette dynamique musicale. Le phonème du port-titre y révèle moins un nom propre avec un signifié défini qu'un espace symbolique, Amsterdam: une terre de déchirure entre l'âme et les dames, donc entre les idéaux et la réalité.

Amsterdam y est un port bien sûr, c'est suffisamment martelé, mais surtout une impasse, où la mélodie élisabéthaine de "Greensleeves » se transfigure en crescendo cosmique. Dans cette fameuse envolée finale, le chanteur belge fantasme les migrations impossibles sur terre ou sur mer, sinon dans les étoiles :

Ils boivent à la santé

Des putains d'Amsterdam

[...] Enfin ils boivent aux dames

$[\ldots]$

Et quand ils ont bien bu

Se plantent le nez au ciel

Se mouchent dans les étoiles

Et ils pissent comme je pleure

Sur les femmes infidèles

Dans le port d'Amsterdam

La chanson orchestre avec maestria le tourbillon d'un surplace : les marins sans navires, sans voyages, atterris, atterrés, tournent et tournent, ils boivent et reboivent. Dans « Amsterdam », leur espace est impasse.

Tel est le décor imaginaire, spatial et atemporel, qui va nous servir de matrice chansonnière, de creuset pour les métissages à venir. 


\section{" Gibraltar », l'espace de la transmutation}

On peut en effet à partir de ce repère observer un déplacement, comme un pont francophone, entre la Belgique et l'Afrique, dans la réécriture d' «Amsterdam » en « Gibraltar » par Abd al Malik.

Ce morceau, paru en 2006 dans un album du même titre, joue de la structure brélienne. Abd al Malik en retourne le sens pour évoquer une figure en miroir de sa propre biographie : cette fois le voyage est possible, et l'Afrique le fantasme lumineux d'une initiation réussie. Il propose ainsi une narration hétérodiégétique pour une dynamique d'initiation autodiégétique. Double déplacement en somme, externe et interne. Et il y fantasme un voyage à rebours de celui qu'ont vécu les immigrants africains, dont il est lui-même un rejeton (né Régis Fayette-Mikano à Paris en 1975, il a grandi à Strasbourg après avoir vécu quatre ans à Brazzaville, auprès de son père haut fonctionnaire congolais). Sauf qu'il s'agit ici d'une dynamique spirituelle plus que réellement migratoire, celle de la rencontre du soufisme que le chanteur a lui-même effectuée en 1999 auprès d'un maître marocain, après son mariage avec la chanteuse Wallen, française née de parents marocains.

Dans « Gibraltar », même l'introduction mime les applaudissements de l'enregistrement brélien à l'Olympia, en 1964. S’y entend également la même répétition lancinante du lieuseuil : "Sur le détroit de Gibraltar » remplace l'anaphore "Dans le port d'Amsterdam », mais cette fois en seuil continental plutôt qu'en limite entre terre et mer. Ce leitmotiv « Dans le port de Gibraltar », souligné par la lancinance des mêmes trois notes sans cesse répétées au clavier, vise moins à laisser imaginer la transposition de Brel ailleurs, que sa prolongation en esprit, dans un autre décor, autour d'une même scansion entre rêves, mourir, naître, puis boire et rire, et pleurer, disséminés dans le morceau d'Abd al Malik :

Sur le détroit de Gibraltar, y a un jeune Noir qui pleure un rêve qui prendra vie, une fois passé Gibraltar.

[...] Sur le détroit de Gibraltar, y a un jeune Noir qui meurt sa vie bête de " gangsta rappeur » mais ...

Sur le détroit de Gibraltar, y a un jeune homme qui va naître, qui va être celui qu'les tours empêchaient d'être.

Sur le détroit de Gibraltar, y a un jeune Noir qui boit, dans ce bar où les espoirs se bousculent, une simple canette de Fanta.

$[\ldots]$

Et ça rit autour de lui, et ça pleure au fond de lui.

Le lyrisme ne propose plus comme chez Brel en 1964 le tableau d'un écartèlement existentiel entre la pesanteur de la chair (le phonème "dame " au bout d'Amster-dam) et les aspirations de l'âme (le phonème qui commence $A m$-sterdam), sur ce bout de terre improbable qu'est un port ; cette fois, c'est une épiphanie qui s'énonce et se chante. Outre Brel, c'est alors Rimbaud et sa «Lettre du Voyant » qui sont convoqués pour une expérience alchimique : «Les 
autres le suivent / Ils veulent être or puisqu'ils sont cuivre. » La pulsation que porte Abd $\mathrm{Al}$ Malik révèle le port comme espace de transit vers l'ailleurs, lieu de la transformation du réel en possible, du présent en cadeau à ouvrir :

Faut rien dire et tout est dit, et soudain ... soudain il s'fait derviche tourneur,

Il danse sur le bar, il danse, il n'a plus peur, enfin il hurle comme un fakir, de la vie devient disciple.

Sur le détroit de Gibraltar y a un jeune Noir qui prend vie, qui chante, dit enfin « je t'aime " à cette vie.

Puis les autres le sentent, le suivent, ils veulent être or puisqu'ils sont cuivre.

Comme ce soleil qui danse, ils veulent se gorger d'étoiles, et déchirer à leur tour cette peur qui les voile.

La danse et les étoiles de Brel deviennent positifs après cette épiphanie chantée. Du coup, si cet autoportrait d'un Noir ancien voyou ("gangsta rappeur ") devenant soufi n'est pas sans composantes autobiographiques, le miroir demeure fantasmatique, le jeu des citations l'atteste. Certes, Abd Al Malik chante au finale :

Du détroit de Gibraltar, un jeune Noir vogue, vogue vers le Maroc tout proche.

Vogue vers ce Maroc qui fera de lui un homme...,

Mais s'il répète presque ad libitum le "merveilleux royaume du Maroc ", c'est bien en France que, dans la réalité de son parcours biographique, s'inscrit sa propre métamorphose. Du Maroc il a en effet épousé le désir, puisque sa femme est elle-même une Française d'origine marocaine. Mais c'est de France et en langue française qu'il met en forme ce rêve d'une émigration à rebours, pour tenter de se retrouver fantasmatiquement. On en reste en définitive à l'incantation lyrique, certes plus efficace que le piteux «Il faut que je rentre chez moi " qui, dans "Ces gens-là », concluait les velléités sans suite du canteur brélien. La dynamique n'est pas nulle, le canteur vogue, mais elle est moins véritablement réalisée qu'intérieure : du "Spleen » baudelairien, le modèle qu'il adopte alors est celui de l'" Invitation au voyage "...

Venu du rap et des banlieues, Abd al Malik marie ainsi Rimbaud, Baudelaire à Brel et au soufisme, et s'apprête à travailler aussi bien avec François Rauber que sur l'œuvre de Camus : sa dynamique fait de l'immigration sa matière même, dialogue fécond de cultures pour un syncrétisme inventif, mais assurément en langue française, cette accueillante matrice.

\section{Stromae, la sublimation du bâtard}

Venu du rap lui aussi, mais de la même nationalité belge que Brel, Stromae, né en 1985 d'une mère flamande et d'un père ruandais, et orphelin à 9 ans de son père victime du génocide ruandais entre Hutus et Tutsis, y fait explicitement écho dans plusieurs chansons : 
«Bâtard ", art poétique du métissage sur lequel je reviendrai, ou «Papaoutai ». Entre Afrique et Europe comme Abd al Malik, Paul Van Haver, devenu le chanteur Stromae, verlan de maestro, tire une énergie dansante de ses douleurs sublimées. Son air de "Papaoutai " parvient à faire danser toute une génération - formidable métamorphose vitale d'une tragédie personnelle et collective, d'un continent à l'autre.

On entend bien sûr, dans ce néologisme " papaoutai " aux sonorités pseudo-primitives (comme un écho à des Papous, ou une île voisine des Touamotou), l'absence d'un père Papa où t'es ? - mais transfigurée par le rythme et la mélodie de la chanson en onomatopée dansante. Pourtant, en creux de cette danse a priori réconfortante s'entend aussi la suggestion d'une duperie fondamentale, "papaoutai " appelant bien sûr le verbe < empapaouter ', que la chanson explicite :

Tout l'monde sait comment on fait des bébés

Mais personne sait comment on fait des papas

L'enjeu est clair pour le fils d'un père mort au Ruanda lorsqu'il avait neuf ans : restaurer une image, combler un manque, ce que l'affirmation positive de la chanson tend à proposer par son rythme, tout en soulignant, car rien n'est simple, que le manque subsiste, selon le principe des balancements ambigus dont l'œuvre est parcourue, comme lorsque le canteur homodiégétique semble accepter la disparition paternelle comme un jeu de cache-cache sans fin :

Ah sacré papa,

Dis-moi où es-tu caché,

Ça doit faire au moins mille fois que j'ai,

Compté mes doigts,

Où t'es papa où t’es ?

Où t'es papa où t'es?

Mais pourtant, l'énergie positive se transmet, malgré cette lézarde originelle, la chanson l'affirme dès le début :

Dites-moi d'où il vient,

Enfin je saurais où je vais,

Maman dit que lorsqu'on cherche bien,

On finit toujours par trouver,

Elle dit qu'il n'est jamais très loin,

Qu'il part très souvent travailler,

Maman dit « travailler c'est bien » 
Il s'agit d'aller. Ça va donc quand même, la chanson nous le fait même danser sur ce refraintitre qu'accompagne la contagieuse et étonnante chaleur d'instruments au son électronique et festif. La question de cette autodiégèse partielle est bien sûr vers où on va sans papa, ce que ne résout pas le clip, où le père reste un mannequin, sauf à un rare moment d'akmé, malgré toutes les danses de son fils, qui ne rejoint finalement son père qu'en se figeant à son tour : la danse hésite ainsi entre le statut de divertissement, celui d'exutoire, ou celui de rituel magique mais éphémère dans ses effets, durant le temps compté de la chanson...

Sans père présent certes, Stromae ne se présente pas pour autant sans origine : un dédoublement résume peut-être tous ceux qu'on rencontre dans son album phare justement titré Racines carrées, au pluriel. C'est l'image du < bâtard ’, retournée en affirmation positive, dans la chanson du même titre. S'y joue en effet un éloge des métissages, en forme de provocation assumée, y compris dans le mélange des genres esthétiques, entre des couplets très marqués par le phrasé du rap, et des refrains chantés, toujours très dansants, selon cette dimension festive qui est l'une des marques de fabrique de Stromae. Le premier couplet commence ainsi, sur un mode de scansion parlée :

T'es de droite ou t'es de gauche?

T'es beauf ou bobo de Paris?

Soit t'es l'un ou soit t'es l'autre

T'es un homme ou bien tu péris

Cultrice ou patéticienne

Féministe ou la ferme

Soit t'es macho, soit homo

Mais t'es phobe ou sexuel

$[\ldots]$

Mais t'es Hutu ou Tutsi ?

Flamand ou Wallon?

Bras ballants ou bras longs?

Finalement t'es raciste?

Mais t'es blanc ou bien t'es marron, hein?

Ni l'un, ni l'autre

Bâtard, tu es, tu l'étais et tu le restes

Le texte, très travaillé, joue des contrepieds, des décalages, des enjambements qui annoncent bien sûr un souci de dépasser les oppositions binaires de la part du chanteur. Ainsi, parvientil brillamment à surmonter la fin que signifie " tu péris ", en transformant, par la grâce d'un enjambement, le verbe en préfixe, lui-même dédoublé pour une sorte de zeugma virtuel, d'abord en un approximatif "péricultrice [sic] " puis en une plausible "péripatéticienne ". Deux façons balancées d'opposer la vie à la mort annoncée. Le même processus se produit 
ensuite pour le préfixe ‘ homo `. Mais plus loin le couplet va au-delà des résolutions de binarités, pour aborder des rives autobiographiques.

Quand le texte balance entre Hutu et Tutsi pour un fils de Ruandais dont le père est mort lors du génocide, l'enjeu résonne avec gravité. La condamnation des oppositions mortiferes se leste des souffrances endurées par l'interprète dans sa propre existence. Métis puisque sa mère est belge, il endosse aussi dans le même élan la querelle des Wallons et des Flamands comme éléments de son patrimoine propre. La question "T'es blanc ou bien t'es marron " sonne donc avec une cruelle ironie pour la couleur de peau du chanteur, mais il l'assume avec la distance que confere la connotation négative de la couleur ' marron ?. Un nègre ' marron ', c'est en effet un noir qui ruse, qui se révolte ou qui ne s'adapte au monde blanc que pour tenter de s'y faire une place. Se retrouver « marron ', c'est aussi s'être fait duper. Le texte de ce premier couplet développe donc une violence qui n'épargne pas le chanteur, dont la distinction d'avec le canteur semble alors poreuse. C'est alors que le phrasé scandé, issu du rap, laisse soudain place à un chant, dont les paroles quatre fois répétées deviennent refrain, mais refrain dansant, tonique et gai, affirmation dynamique d'une identité qui transcende toutes les étiquettes et s'envole sur des sons électros :

Ni l'un ni l'autre, je suis, j'étais, et resterai moi

Le balancement comme évidence dynamique s'y traduit à la fois par l'envolée lyrique dans le chant, mais aussi par la prise de parole à la première personne du canteur : son < je > enfin débridé y répond à la litanie des adresses à la seconde personne, ces < tu > que martèlent les couplets comme autant d'attaques à l'hybridité. La revendication de pureté raciale, il ne faut pas l'oublier, était précisément celle qui justifiait pour ses auteurs les crimes génocidaires au Ruanda.

Être soi et être bâtard s'affichent donc en synonymes positifs, revendiqués, sublimés en air à propager, d'autant mieux proposés en refrain qu'ils en deviennent étendard universel, à répéter et danser en chœur, et bien évidemment à partager et à faire partager par le biais d'un chant collectif et joyeux. Les cicatrices de Paul Van Haver ne sont pas effacées, elles sont transfigurées par la dynamique et l'esthétique de Stromae.

Stromae, ce nom en verlan pour inverser le maestro, pour avoir été un jeune orphelin et un métis transcontinental, s'avère maître en résilience, acrobate du corps, des rythmes et des mots, capable de plus étonnants rétablissements, balançant en permanence pour chanter l'énergie des résurrections.

Ainsi, d'Afrique à l'Europe, chez ces deux chanteurs, l'inspiration migratoire sait dépasser la chronique des drames pour suggérer le jubilatoire de métissages aboutis, de la musique élisabéthaine à Brel en passant par Rimbaud, ou le débridement des mots-valises, des mots hybrides. L'immigration se transfigure en la matière même du chant, tant par le tissage/métissage des référents multiples et croisés, que par la dynamique de l'alchimie heureuse dont 
les chansons sont le creuset. Le teint cuivré s'y révèle mine d'or. Chez eux, immigrer s'avère tressage fécond.

Aussi, fort de cette réussite chansonnière, et parodiant Georgius, j'oserais alors conclure sur un mot forcément valise, puisqu'il s'agit de métisser. Si, pour chanter l'immigration à l'œuvre en francophonie, l'art du bâtard s'avérait le plus bath des arts d'aujourd'hui, serait-il donc venu, le temps du bath-art?

\section{Notes}

1 Stéphane Hirschi est professeur à l'Université Polytechnique Hauts de France (Valenciennes), où il dirige l'axe «Transports et mobilités » au sein du laboratoire CALHISTE.

\section{Bibliographie}

Brunschwig, Chantal / Calvet, Louis-Jean / Klein, Jean-Claude : Cent ans de chanson française. Paris : Seuil, 1981.

Clouzet, Jean : Jacques Brel. Paris : Seghers, 1964 (éd. augmentée 1982).

Hirschi, Stéphane : Jacques Brel. Chant contre silence. Paris : Nizet, 1995.

Hirschi, Stéphane : Chanson : l'art de fixer l'air du temps - de Béranger à Mano Solo. Paris : Les Belles Lettres/PUV, 2008.

Hirschi, Stéphane : "Le pays du désir francophone : topique d'un chaud-froid, de Brel à Abd al Malik ». In : Hirschi, Stéphane / Huftier, Arnaud (éds) : Le froid et le chaud ou les glaces d'Haïti : autour de René Depestre, horizons d'un lyrisme francophone? Amiens : Éd. Encrage, 2012, 219-236.

Hirschi, Stéphane : La chanson française depuis 1980 - de Goldman à Stromae, entre vinyles et MP3. Paris : Les Belles Lettres/PUV, 2016.

Hirschi, Stéphane : «Le métissage musical - autour de Bernard Lavilliers ». In : Les enfants de la Zique 22 (2016), https://www.reseau-canope.fr/les-enfants-de-la-zique (consultation 01.03.2018).

\section{Discographie}

Abd Al Malik : «Gibraltar » (2006). In : https://www.youtube.com/watch?v=AX0y5tkPHgM (consultation 01.03.2018).

Baker, Joséphine : «Ma petite Tonkinoise» (1931). In : https://www.youtube.com/watch?v=iGr3c1dCm74 (consultation 01.03.2018).

Brel, Jacques : «Amsterdam» (1964). In : https://www.youtube.com/watch?v=Fcw4SRGcY_E (consultation 01.03.2018).

Ferré, Léo : «Rotterdam » (1970). In : https://www.youtube.com/watch?v=CAkq3KGgV7E (consultation 01.03.2018). 
Gainsbourg, Serge : «Aux armes et cætera » (1979). In : http://www.dailymotion.com/video/xur3m0_ serge-gainsbourg-aux-armes-et-caetera_music (consultation 01.03.2018).

Mayol, Félix : «À la cabane bambou » (1899). In : https://www.youtube.com/watch?v=RcYli1cOnOY (consultation 01.03.2018).

Montero, Germaine : "Tha-ma-ra-boum-di-hé ». In : Montero, Germaine : La belle époque. Le chant du monde LD-M-4013, 1953 (33 tours).

Polin : «Ma petite Tonkinoise » (1906). In : https://www.youtube.com/watch?v=mMEGSbqSZ_M (consultation 01.03.2018).

Nougaro, Claude : «Le jazz et la java » (1962). In : https://www.youtube.com/watch?v=PM5Ohz4WaKA (consultation 01.03.2018).

Stromae : «Papaoutai » (2013). In : https://www.youtube.com/watch?v=oiKj0Z_Xnjc (consultation 01.03.2018).

Stromae : «Bâtard» (2013). In : https://www.youtube.com/watch?v=fatfDUPiJ5U (consultation 01.03.2018).

Vassiliu, Pierre : «Qui c'est celui-là » (1974). In : https://www.youtube.com/watch?v=GNWKWhdnRDI (consultation 01.03.2018). 